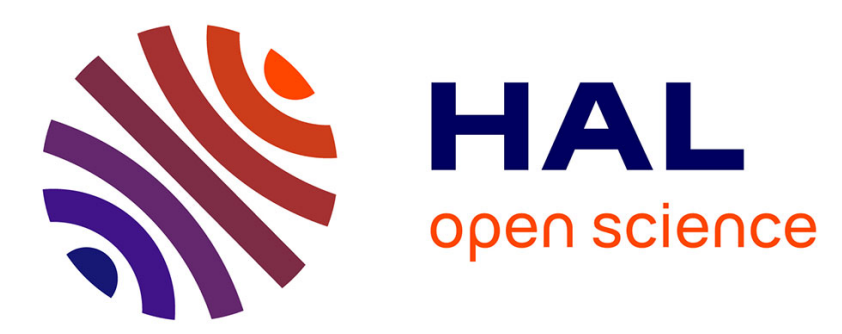

\title{
Output synchronization of unknown heterogeneous agents via distributed model reference adaptation
}

Simone Baldi, Shuai Yuan, Paolo Frasca

\section{To cite this version:}

Simone Baldi, Shuai Yuan, Paolo Frasca. Output synchronization of unknown heterogeneous agents via distributed model reference adaptation. IEEE Transactions on Control of Network Systems, 2019, 6 (2), pp.515-525. 10.1109/TCNS.2018.2844260 . hal-01809667

\section{HAL Id: hal-01809667 https://hal.science/hal-01809667}

Submitted on 28 Jun 2018

HAL is a multi-disciplinary open access archive for the deposit and dissemination of scientific research documents, whether they are published or not. The documents may come from teaching and research institutions in France or abroad, or from public or private research centers.
L'archive ouverte pluridisciplinaire HAL, est destinée au dépôt et à la diffusion de documents scientifiques de niveau recherche, publiés ou non, émanant des établissements d'enseignement et de recherche français ou étrangers, des laboratoires publics ou privés. 


\title{
Output synchronization of unknown heterogeneous agents via distributed model reference adaptation
}

\author{
Simone Baldi, Member, IEEE, Shuai Yuan and Paolo Frasca, Member, IEEE
}

\begin{abstract}
This work presents a distributed model reference adaptive methodology for output synchronization of heterogeneous linear agents with unknown dynamics. We consider a setting in which the control input is communicated among neighbors, instead of observer variables. For those agents that can access the signals of the reference model, classical model reference adaptation laws lead to leader synchronization; for those agents that cannot access such signals, synchronization must be achieved by taking the neighboring agents as an alternative reference model. We show that these two groups of agents give rise to two types of matching conditions: the standard conditions to match the reference model, and new distributed matching conditions among neighboring agents. Since all matching gains are unknown, the gains are adapted online via Lyapunov-based estimation. Asymptotic synchronization is proven analytically, and numerical examples show the effectiveness of the approach.
\end{abstract}

Index Terms-Output synchronization, distributed adaptive control, heterogeneous unknown agents.

\section{INTRODUCTION}

$\mathbf{I}$ $\mathrm{N}$ recent years, cooperative control of multiagent systems has received the attention of many scientific communities, due to its impact in formation flying, smart energy, smart traffic and other crucial areas [1]-[5]. An important problem in cooperative control is to achieve in a distributed way (i.e. using local information) a common behavior for the entire network: this is the so-called synchronization problem [6], [7], sometimes referred to as the consensus problem [8]-[11] when the behavior to be achieved is a constant. Synchronization serves as a building block for more sophisticated coordination tasks [12]: popular forms of synchronization are based on creating homogeneous dynamics (homogenization), e.g. by endowing all agents with an internal model of the common behavior to be achieved [13], [14], or by matching some desired dynamics [15], [16]. In literature we can distinguish at least two families of protocols through which synchronization can be implemented. In the first family, the agents that are not connected to the leader generating the reference signal will construct an observation of such signal via a distributed observer [13], [17]. In the second family, a distributed input is used in place of the distributed observer: in other words, communication of the control input is adopted in place of communicating the observer variables [18]-[20]. Advances in the distributed input approach include stability of the interconnected system

S. Baldi and S. Yuan are with Delft Center for Systems and Control, Delft University of Technology, The Netherlands. S. Yuan is also with School of Astronautics, Harbin Institute of Technology, China. P. Frasca is with Univ Grenoble Alpes, CNRS, Inria, Grenoble INP (Institute of Engineering Univ. Grenoble Alpes), GIPSA-Lab, France. E-mails: s.baldi@tudelft.nl, s.yuan1@tudelft.nl, paolo.frasca@gipsa-lab.fr. The work has been partially funded by EC, FP7-ICT-2013.3.4, under contract \#611538 (LOCAL4GLOBAL)
[21], or addressing the presence of switching communication topologies [22]. Variants to this strategy include discontinuous strategies based on sliding mode [23], [24].

Synchronizing solutions have been proposed when the agents are uncertain but homogeneous [25], [26], heterogeneous with no uncertainty [27], [28], or heterogeneous with specific structural uncertainty [29]. Despite these results, synchronization in the joint presence of heterogeneity and uncertainty is still a major problem. Using the homogenization framework, the idea is to cancel the effects of heterogeneity and/or uncertainty via feedback gains: up to now, non-adaptive (i.e. fixed-gain) feedback with possibly adaptive coupling weights has been mainly explored [30]-[32]. However, it is well known that as uncertainty grows bigger and bigger no fixed-gain feedback may cope with it [33]. Therefore, it is of fundamental importance to integrate adaptive control methodologies in cooperative control, so that teams of agents can respond to parametric changes that might occur while the cooperative task is carried out. Output synchronization in the joint presence of heterogeneity and uncertainty has been proposed, with distributed observer protocols, for the special class of Euler-Lagrange agents [34], [35]: however, no adaptive output synchronization protocols have been reported using distributed input protocols, which motivates this work.

The main contribution of this work is an adaptive methodology based on distributed input protocol for output synchronization of heterogeneous agents with linear and unknown dynamics. A distributed model reference adaptive control framework is used to solve the problem. For the agents with direct access to the leader signals, the classical reference model idea is adopted; for the agents with access to the leader signals a new reference model is defined by the neighboring agents to which each agent aims to converge. Homogenization of the two groups of agents gives rise to two types of matching conditions: the classical matching conditions between an agent and the reference model, and new distributed matching conditions among neighboring agents. Since all matching gains are unknown (in view of the unknown dynamics), we design a Lyapunov-based adaptation to handle possibly large uncertainties. Asymptotic synchronization error is shown analytically on acyclic graphs, and extensions to general graphs are discussed both analytically and via numerical examples.

The paper is organized as follows: Sect. II formulates the problem and Sect. III recalls the standard matching conditions for model reference synchronization. Sect. IV introduces the new distributed matching conditions for those agents not connected to the reference model. Sect. V contains the main synchronization, Sect. VI demonstrates the findings via simulations and Sect. VII concludes the work. 
Notation: The transpose and Euclidean norm of a (column) vector are indicated with $x^{T}$ and $\|x\|$, respectively. A signal $x: \mathbb{R} \rightarrow \mathbb{R}^{n}$ belongs to $\mathscr{L}_{2}$ class $\left(x \in \mathscr{L}_{2}\right)$ if $\int_{0}^{t}\|x(\tau)\|^{2} \mathrm{~d} \tau<\infty$, $\forall t \geq 0$, and to $\mathscr{L}_{\infty}$ class $\left(x \in \mathscr{L}_{\infty}\right)$, if $\max _{t \geq 0}\|x(t)\|<\infty, \forall t \geq 0$. A directed graph (digraph) is indicated with the pair $(\mathscr{N}, \mathscr{E})$, where $\mathscr{N}$ is a finite set of nodes, and $\mathscr{E} \in \mathscr{N} \times \mathscr{N}$ is a set of ordered pair of nodes, called edges. The adjacency matrix $\mathscr{A}=\left[a_{i j}\right]$ of a weighted digraph is defined as $a_{i i}=0$ and $a_{i j}>0$ if $(j, i) \in \mathscr{E}$, where $i \neq j$, while the Laplacian matrix is defined as $\mathscr{L}=\left[l_{i j}\right]$, where $l_{i i}=\sum_{j} a_{i j}$ and $l_{i j}=-a_{i j}$, if $i \neq j$.

\section{PROBLEM FORMULATION}

For ease of presentation, let us start by formulating a simple synchronization problem for two agents, denoted with subscripts 1 and 2. Omitting the time index $t$ for brevity, their dynamics are expressed in the transfer function ${ }^{1}$ form

$$
\begin{aligned}
& y_{1}=G_{1}(s) u_{1}=k_{1} \frac{Z_{1}(s)}{R_{1}(s)} u_{1} \\
& y_{2}=G_{2}(s) u_{2}=k_{2} \frac{Z_{2}(s)}{R_{2}(s)} u_{2}
\end{aligned}
$$

where $u_{1}, u_{2} \in \mathbb{R}$ and $y_{1}, y_{2} \in \mathbb{R}$ are the inputs and the outputs of the two agents; the polynomials $Z_{1}(s), Z_{2}(s)$ and $R_{1}(s)$, $R_{2}(s)$ are unknown monic polynomials and $k_{1}, k_{2}$ are unknown constants referred to as the high frequency gains.

Let us assume a directed connection from agent 1 to agent 2 , i.e. the corresponding digraph is described by $\mathscr{N}=\{1,2\}$, $\mathscr{E}=\{(1,2)\}$. Let us also introduce the connection of agent 1 to a reference model (leader) denoted with the subscript 0

$$
y_{0}=G_{0}(s) r=k_{0} \frac{Z_{0}(s)}{R_{0}(s)} r
$$

where $r \in \mathbb{R}, y_{0} \in \mathbb{R}$ are the reference and the output of the reference model. The polynomials $Z_{0}(s), R_{0}(s)$ are known monic polynomials and $k_{0}$ is the known high frequency gain. As agent 1 is the only agent that can access the reference input $r$, let us consider the following problem:

Synchronization to the reference model for two agents: Find a distributed synchronization protocol that achieves, for any bounded $r$ and using only local measurements, the following tasks: for agent 1 , synchronize to the output of the reference model, i.e. $y_{1} \rightarrow y_{0}$ for $t \rightarrow \infty$; for agent 2 (which cannot synchronize to agent 0 directly) synchronize to the output of agent 1, i.e. $y_{2} \rightarrow y_{1}$ for $t \rightarrow \infty$, which implies $y_{2} \rightarrow y_{0}$.

To meet the synchronization objectives, we need some wellposedness assumptions, classical in adaptive control [37]. Agent assumptions:

A1. $Z_{i}(s), i \in\{1,2\}$, are monic Hurwitz polynomials;

A2. An upper bound $n$ to the degree $n_{i}$ of $R_{i}(s), i \in\{1,2\}$, is known;

A3. The relative degree $n^{*}=n_{i}-m_{i}$ of $G_{i}(s), i \in\{1,2\}$, is known, where $m_{i}$ is the degree of the numerator $Z_{i}(s)$;

A4. The signs of the high frequency gains $k_{i}, i \in\{1,2\}$, are known.

\footnotetext{
${ }^{1}$ We consider the single-input single-output case: multivariable extension is possible via appropriate canonical forms [36], which are not considered here to avoid technicalities that might hide the key ideas behind the approach.
}

Reference model assumptions:

M1. $Z_{0}(s), R_{0}(s)$ are monic Hurwitz polynomials;

M2. The relative degree of $G_{0}(s)$ is also $n^{*}$.

Assumption for ease of presentation:

E1. The relative degree is $n^{*}=1$ and $G_{0}(s)$ is strictly positive $\mathrm{real}^{2}$ (SPR).

Remark 1. Assumptions A1-A4 and M1-M2 stem from the model reference adaptive control framework used to achieve synchronization [37]. Assumption A1 requires the agents to be minimum phase, while M1 obviously imposes the reference model to be open-loop stable. Assumptions A2, A3 and M2 are required to define the degree of the control law, while A4 determines the sign of the adaptation law. Assumption E1 gives a straightforward Lyapunov analysis which makes the presentation of the design easier: extensions to $n^{*}>1$ and non strictly positive real $G_{0}(s)$ can be obtained as discussed in [38, Sect. 6.4.2 and 6.4.3].

\section{HoMOGENIZATION VIA MATCHING CONDITIONS}

If an agent $j$ can access the reference input $r$, synchronization among agents 0 and $j$ is known in literature as model reference adaptive control [37]: the approach (here briefly recalled) passes through a control law in the form

$$
a_{0 j} u_{j}=a_{0 j} l_{j}^{* T} \frac{\alpha(s)}{\Lambda(s)} u_{j}+a_{0 j} f_{j}^{* T} \frac{\alpha(s)}{\Lambda(s)} y_{j}+a_{0 j} g_{j}^{*} y_{j}+a_{0 j} c_{j}^{*} r
$$

where $a_{0 j}$ is the weighted connection between agents $j$ and 0 (the term $a_{0 j}$ in (3) has not been simplified in order to be consistent with the next sections where multiple connections with other agents might be present). In (3), $\Lambda(s)$ is a Hurwitz monic polynomial to be designed as $\Lambda(s)=\Lambda_{0}(s) Z_{0}(s)$, with $\Lambda_{0}(s)$ Hurwitz: $\alpha(s)$ is a vector defined as

$$
\begin{aligned}
& \alpha(s)=\left[\begin{array}{lllll}
s^{n-2} & s^{n-3} & \ldots & s & 1
\end{array}\right]^{T} \quad \text { for } n \geq 2 \\
& \alpha(s)=0 \quad \text { for } n=1 \text {. }
\end{aligned}
$$

The next step is to find the conditions under which a control law in the form (3) makes agent $j$ to match the reference model (2), i.e. to have its same transfer function

$$
\frac{y_{j}(s)}{r(s)}=k_{0} \frac{Z_{0}(s)}{R_{0}(s)} \text {. }
$$

Such conditions are obtained by writing $y_{j}$ in closed loop

$$
\begin{aligned}
y_{j} & =k_{j} \frac{Z_{j}(s)}{R_{j}(s)}\left(l_{j}^{* T} \frac{\alpha(s)}{\Lambda(s)} u_{j}+f_{j}^{* T} \frac{\alpha(s)}{\Lambda(s)} y_{j}+g_{j}^{*} y_{j}+c_{j}^{*} r\right) \\
\frac{y_{j}(s)}{r(s)} & =\frac{\frac{c_{j}^{*} k_{j} Z_{j}(s)}{R_{j}(s)}}{\left(1-\frac{l_{j}^{* T} \alpha(s)}{\Lambda(s)}-\frac{f_{j}^{* T} \alpha(s)}{\Lambda(s)} \frac{k_{j} Z_{j}(s)}{R_{j}(s)}-g_{j}^{*} \frac{k_{j} Z_{j}(s)}{R_{j}(s)}\right)} .
\end{aligned}
$$

By comparing the transfer functions (2) and (6) we derive the matching conditions between agent $j$ and the reference model:

$$
\begin{gathered}
R_{j}(s)\left(\Lambda(s)-l_{j}^{* T} \alpha(s)\right)-k_{j} Z_{j}(s)\left(f_{j}^{* T} \alpha(s)+g_{j}^{*} \Lambda(s)\right) \\
=Z_{j}(s) R_{0}(s) \Lambda_{0}(s)
\end{gathered}
$$

\footnotetext{
${ }^{2}$ A strictly positive-real rational function $G(s)$ is real when $s$ is real and has strictly positive real part when $s$ has a strictly positive real part.
} 
and $c_{j}^{*}=k_{0} / k_{j}$. The matching condition (7) and control law (3) present two problems: the first one is that solution of (7) requires the knowledge of the polynomials $Z_{j}(s), R_{j}(s)$ and of the constants $k_{j}$, which are all unknown. The second problem is that, even if all parameters were perfectly known, (3) can be implemented only by the agents with access to $r$. In the rest of this section we see how to overcome the first problem, while in Section IV we address the second problem.

With reference to our network with two agents 1 and 2, as the gains $l_{1}^{*}, f_{1}^{*}, g_{1}^{*}$, and $c_{1}^{*}$ for the controller (3) of agent 1 are unknown, let us use

$$
a_{01} u_{1}=a_{01} l_{1}^{T} \frac{\alpha(s)}{\Lambda(s)} u_{1}+a_{01} f_{1}^{T} \frac{\alpha(s)}{\Lambda(s)} y_{1}+a_{01} g_{1} y_{1}+a_{01} c_{1} r
$$

where $l_{1}, f_{1}, g_{1}$, and $c_{1}$ are estimates for $l_{1}^{*}, f_{1}^{*}, g_{1}^{*}$, and $c_{1}^{*}$ respectively, derived via the following well-known adaptive control result.

Result 1. [37] Under Assumptions A1-A4, M1-M2 and E1, the following adaptive law

$$
\begin{aligned}
\dot{\omega}_{u_{1}} & =F \omega_{u_{1}}+d u_{1} \quad \dot{\omega}_{y_{1}}=F \omega_{y_{1}}+d y_{1} \\
a_{01} u_{1} & =\theta_{1}^{T} \omega_{1} \\
\dot{\theta}_{1} & =-\Gamma a_{01} e_{10} \omega_{1} \operatorname{sgn}\left(\frac{k_{1}}{k_{0}}\right)
\end{aligned}
$$

where $e_{10}=y_{1}-y_{0}, \Gamma>0$ and

$$
\omega_{1}=\left[\begin{array}{c}
a_{01} \omega_{u_{1}} \\
a_{01} \omega_{y_{1}} \\
a_{01} y_{1} \\
a_{01} r
\end{array}\right], \quad F=\left[\begin{array}{ccc}
-\lambda_{n-2} & \ldots & -\lambda_{0} \\
I_{n-2} & & 0
\end{array}\right], \quad d=\left[\begin{array}{c}
1 \\
0 \\
\vdots \\
0
\end{array}\right]
$$

and $\lambda_{i}$ are the coefficient of

$$
\Lambda(s)=s^{n-1}+\lambda_{n-2} s^{n-2}+\ldots+\lambda_{1} s+\lambda_{0}
$$

guarantees $y_{1} \rightarrow y_{0}$ for $t \rightarrow \infty$

Proof. A sketch of the proof is recalled, which will be useful to understand the main synchronization result. Since $(F, d)$ is a state-space realization of $\frac{\alpha(s)}{\Lambda(s)}$, the adaptive law (9) is a statespace realization of controller (8) with

$$
\theta_{1}=\left[\begin{array}{llll}
l_{1}^{T} & f_{1}^{T} & g_{1} & c_{1}
\end{array}\right]^{T} .
$$

Let us take an analogous state-space realization of the ideal control law (3)

$$
a_{01} u_{1}^{*}=\theta_{1}^{* T} \omega_{1}
$$

where $\theta_{1}^{*}=\left[\begin{array}{llll}l_{1}^{* T} & f_{1}^{* T} & g_{1}^{*} & c_{1}^{*}\end{array}\right]^{T}$. Adopting a state-space realization of agent 1

$$
\begin{aligned}
& \dot{x}_{1}=A_{1} x_{1}+b_{1} u_{1} \\
& y_{1}=h_{1}^{T} x_{1}
\end{aligned}
$$

and after augmenting the state $x_{1}$ with the controller states, $\bar{x}_{1}=\left[\begin{array}{lll}x_{1}^{T} & \omega_{u_{1}}^{T} & \omega_{y_{1}}^{T}\end{array}\right]^{T}$, we obtain

$$
\begin{aligned}
& \dot{\bar{x}}_{1}=\bar{A}_{1} \bar{x}_{1}+\bar{B}_{1} c_{1}^{*} r+\bar{B}_{1}\left(u_{1}-\theta_{1}^{* T} \bar{\omega}_{1}\right) \\
& y_{1}=\bar{C}_{1}^{T} \bar{x}_{1}
\end{aligned}
$$

with $\bar{\omega}_{1}=\omega_{1} / a_{01}$ and

$$
\begin{aligned}
& \bar{A}_{1}=\left[\begin{array}{ccc}
A_{1}+b_{1} g_{1}^{*} h_{1}^{T} & b_{1} l_{1}^{* T} & b_{1} f_{1}^{* T} \\
d g_{1}^{*} h_{1}^{T} & F+d l_{1}^{* T} & d f_{1}^{* T} \\
d h_{1}^{T} & 0 & F
\end{array}\right], \\
& \bar{B}_{1}=\left[\begin{array}{c}
b_{1} \\
d \\
0
\end{array}\right], \quad \bar{C}_{1}^{T}=\left[\begin{array}{lll}
h_{1}^{T} & 0 & 0
\end{array}\right] .
\end{aligned}
$$

Let us now take the following non-nominal state-space representation of the reference model

$$
\begin{aligned}
& \dot{\bar{x}}_{0}=\bar{A}_{1} \bar{x}_{0}+\bar{B}_{1} c_{1}^{*} r \\
& y_{0}=\bar{C}_{1}^{T} \bar{x}_{0} .
\end{aligned}
$$

After defining $\tilde{x}_{10}=\bar{x}_{1}-\bar{x}_{0}$, we obtain the error dynamics

$$
\begin{aligned}
& \dot{\tilde{x}}_{10}=\bar{A}_{1} \tilde{x}_{10}+\bar{B}_{1}\left(u_{1}-\theta_{1}^{* T} \bar{\omega}_{1}\right) \\
& e_{10}=\bar{C}_{1}^{T} \tilde{x}_{10}
\end{aligned}
$$

which become, after substituting (8) into the (18)

$$
\begin{aligned}
& \dot{\tilde{x}}_{10}=\bar{A}_{1} \tilde{x}_{10}+\overline{\bar{B}}_{1} \rho_{1}^{*} \tilde{\theta}_{1}^{T} \bar{\omega}_{1} \\
& e_{10}=\bar{C}_{1}^{T} \tilde{x}_{10}
\end{aligned}
$$

where $\overline{\bar{B}}_{1}=\bar{B}_{1} c_{1}^{*}, \rho_{1}^{*}=\frac{1}{c_{1}^{*}}$, and $\tilde{\theta}_{1}=\theta_{1}-\theta_{1}^{*}$. In addition, it can be verified that [38, Sect. 6.3.2, eq. (6.3.25)]

$$
\bar{C}_{1}\left(s I-\bar{A}_{1}\right)^{-1} \overline{\bar{B}}_{1}=G_{0}(s)
$$

As $G_{0}(s)$ is taken to be strictly positive-real from E1, we can consider the Lyapunov function

$$
V_{1}\left(\tilde{\theta}_{1}, \tilde{x}_{10}\right)=a_{01}^{2} \frac{\tilde{x}_{10}^{T} P \tilde{x}_{10}}{2}+\frac{\tilde{\theta}_{1}^{T} \Gamma^{-1} \tilde{\theta}_{1}}{2}\left|\rho_{1}^{*}\right|
$$

where $P=P^{T}>0$ satisfies the Kalman-Yakubovich Lemma

$$
\begin{aligned}
P \bar{A}_{1}+\bar{A}_{1}^{T} P & =-q q^{T}-v L \\
P \overline{\bar{B}}_{1} & =\bar{C}_{1}
\end{aligned}
$$

with $L=L^{T}>0$, and $v>0$. The time derivative of $V_{1}$ is

$$
\begin{aligned}
\dot{V}_{1}= & -a_{01}^{2} \frac{\tilde{x}_{10}^{T} q q^{T} \tilde{x}_{10}}{2}-a_{01}^{2} \frac{v}{2} \tilde{x}_{10}^{T} L \tilde{x}_{10} \\
& +a_{01} \tilde{x}_{10}^{T} P \overline{\bar{B}}_{1} \rho_{1}^{*} \tilde{\theta}_{1}^{T} \omega_{1}+\tilde{\theta}_{1}^{T} \Gamma^{-1} \dot{\tilde{\theta}}_{1}\left|\rho_{1}^{*}\right|
\end{aligned}
$$

since $\tilde{x}_{10}^{T} P \overline{\bar{B}}_{1}=\tilde{x}_{10}^{T} \bar{C}_{1}=e_{10}$ and $\rho_{1}^{*}=\left|\rho_{1}^{*}\right| \operatorname{sgn}\left(\rho_{1}^{*}\right)$, we can delete the last two terms by choosing

$$
\dot{\tilde{\theta}}_{1}=\dot{\theta}_{1}=-\Gamma a_{01} e_{10} \omega_{1} \operatorname{sgn}\left(\rho_{1}^{*}\right)
$$

which leads to

$$
\dot{V}_{1}=-a_{01}^{2} \frac{\tilde{x}_{10}^{T} q q^{T} \tilde{x}_{10}}{2}-a_{01}^{2} \frac{v}{2} \tilde{x}_{10}^{T} L \tilde{x}_{10} .
$$

From (25) we obtain that $V_{1}$ has a finite limit, and therefore $\tilde{x}_{10}, \tilde{\theta}_{1} \in \mathscr{L}_{\infty}$. Because $\tilde{x}_{10}=\bar{x}_{1}-\bar{x}_{0} \in \mathscr{L}_{\infty}$ and $\bar{x}_{0} \in \mathscr{L}_{\infty}$, we have $\bar{x}_{1} \in \mathscr{L}_{\infty}$. This implies $x_{1}, y_{1}, \omega_{1} \in \mathscr{L}_{\infty}$. Since from (25) we can establish that $\dot{V}_{1}$ has bounded integral, we have $\tilde{x}_{10}, e_{10} \in \mathscr{L}_{2}$. Furthermore using $\theta_{1}, \omega_{1}, \tilde{x}_{10} \in \mathscr{L}_{\infty}$ in (19) we have $e_{10}, \dot{e}_{10} \in \mathscr{L}_{\infty}$. From Barbalat's Lemma, this implies $e_{10} \rightarrow 0$ for $t \rightarrow \infty$, which concludes the proof. 


\section{DistRibuted MATCHING CONDITIONS}

The control law (8) with adaptation (9) removes the problem of unknown $l_{1}^{*}, f_{1}^{*}, g_{1}^{*}$ and $c_{1}^{*}$ : yet, a problem remains as the control law (8) is implementable only for those agents with access to $r$. To overcome this problem, we will derive new (distributed) matching conditions between neighboring agents $j$ and $i$. The following proposition is given.

Proposition 1. Let us consider an agent $j$ not connect to the reference model. For a control law in the form

$$
\begin{aligned}
\sum_{i=1}^{N} a_{i j} u_{j}= & \sum_{i=1}^{N} a_{i j} l_{j i}^{* T} \frac{\alpha(s)}{\Lambda(s)} u_{i}+\sum_{i=1}^{N} a_{i j} f_{j i}^{* T} \frac{\alpha(s)}{\Lambda(s)} y_{i}+\sum_{i=1}^{N} a_{i j} g_{j i}^{*} y_{i} \\
& +\sum_{i=1}^{N} a_{i j} c_{j i}^{*} u_{i}+l_{j}^{* T} \frac{\alpha(s)}{\Lambda(s)} \sum_{i=1}^{N} a_{i j}\left(u_{j}-u_{i}\right) \\
& +f_{j}^{* T} \frac{\alpha(s)}{\Lambda(s)} \sum_{i=1}^{N} a_{i j}\left(y_{j}-y_{i}\right)+g_{j}^{*} \sum_{i=1}^{N} a_{i j}\left(y_{j}-y_{i}\right)
\end{aligned}
$$

the matching conditions between agents $j$ and $i$ are

$$
\begin{gathered}
R_{i}(s)\left(\Lambda(s)-\left(\bar{l}_{j}^{* T}-\bar{l}_{j i}^{* T}\right) \alpha(s)\right)-k_{j} Z_{i}(s)\left(\left(f_{j}^{* T}-f_{j i}^{* T}\right) \alpha(s)\right. \\
\left.+\left(g_{j}^{*}-g_{j i}^{*}\right) \Lambda(s)\right)=Z_{i}(s) R_{0}(s) \Lambda_{0}(s)
\end{gathered}
$$

with

$$
c_{j i}^{*}=\frac{k_{i}}{k_{j}}, \quad \bar{l}_{j i}^{*}=\frac{l_{j i}^{*}}{c_{j i}^{*}}, \quad \bar{l}_{j}^{*}=\frac{l_{j}^{*}}{c_{j i}^{*}} .
$$

Proof. The proof is carried out for agents 1 and 2, without loss of generality. Rewrite (26) to isolate $u_{2}$, as follows

$$
\begin{aligned}
u_{2}= & \frac{\left(l_{21}^{*}-l_{2}^{*}\right)^{T} \alpha u_{1}+\left(f_{21}^{*}-f_{2}^{*}\right)^{T} \alpha y_{1}}{\left(\Lambda-l_{2}^{* T} \alpha\right)} \\
& +\frac{\Lambda\left(g_{21}^{*}-g_{2}^{*}\right) y_{1}+l_{2}^{*} \alpha y_{2}+g_{2}^{*} y_{2} \Lambda+c_{21} u_{1} \Lambda}{\left(\Lambda-l_{2}^{* T} \alpha\right)} .
\end{aligned}
$$

Substitute (29) into (1) to get

$$
\begin{aligned}
y_{2}= & k_{2} \frac{Z_{2}}{R_{2}}\left[\frac{\left(l_{21}^{*}-l_{2}^{*}\right)^{T} \alpha u_{1}+\left(f_{21}^{*}-f_{2}^{*}\right)^{T} \alpha y_{1}}{\left(\Lambda-l_{2}^{* T} \alpha\right)}\right. \\
& \left.+\frac{\Lambda\left(g_{21}^{*}-g_{2}^{*}\right) y_{1}+l_{2}^{*} \alpha y_{2}+g_{2}^{*} y_{2} \Lambda+c_{21} u_{1} \Lambda}{\left(\Lambda-l_{2}^{* T} \alpha\right)}\right]
\end{aligned}
$$

and rearrange (30) as

$$
\begin{aligned}
& \left(R_{2}(s)\left(\Lambda(s)-l_{2}^{* T} \alpha(s)\right)-k_{2} Z_{2}(s)\left(l_{2}^{* T} \alpha(s)+\Lambda(s) g_{2}\right)\right) y_{2} \\
& \quad-\left(k_{2} Z_{2}(s)\left(\left(f_{21}^{*}-f_{2}^{*}\right)^{T} \alpha(s)+\Lambda(s)\left(g_{21}^{*}-g_{2}^{*}\right)\right)\right) y_{1} \\
& \quad=k_{2} Z_{2}(s)\left(\Lambda(s) c_{21}^{*}+\left(\left(l_{21}^{*}-l_{2}^{*}\right)^{T} \alpha(s)\right) u_{1} .\right.
\end{aligned}
$$

At the same time, applying (7) to agent 2 leads to

$$
\begin{aligned}
& \left(Z_{2} \Lambda_{0} R_{0}\right) y_{2}+k_{2} Z_{2}\left(f_{2}^{* T} \alpha+\Lambda g_{2}^{*}\right) y_{1} \\
& \quad-k_{2} Z_{2}\left(f_{21}^{* T} \alpha+\Lambda g_{21}^{*}\right) y_{1}=k_{2} Z_{2}\left(\Lambda c_{21}^{*}+\left(\left(l_{21}^{*}-l_{2}^{*}\right)^{T} \alpha\right) u_{1}\right.
\end{aligned}
$$

and

$$
\begin{gathered}
\left(Z_{2}(s) \Lambda_{0}(s) R_{0}(s)\right)\left(y_{2}-y_{1}\right)+\left(R_{2}(s)\left(\Lambda(s)-l_{2}^{* T} \alpha(s)\right)\right. \\
\left.\quad-k_{2} Z_{2}(s)\left(f_{21}^{* T} \alpha(s)+\Lambda(s) g_{21}^{*}\right)\right) y_{1} \\
=c_{21}^{*} k_{2} Z_{2}(s)\left(\Lambda(s)+\frac{l_{21}^{* T}}{c_{21}^{*}} \alpha(s)-\frac{l_{2}^{* T}}{c_{21}^{*}} \alpha(s)\right) u_{1} .
\end{gathered}
$$

Let us define $\bar{l}_{21}^{*}=\frac{l_{21}^{* T}}{c_{21}^{*}}$ and $\bar{l}_{2}^{*}=\frac{l_{2}^{* T}}{c_{21}^{*}}$. Using the least common multiplier between (33) and $G_{1}(s)$, we can rewrite (33) as

$$
\begin{aligned}
& Z_{1}\left(Z_{2} \Lambda_{0} R_{0}\right)\left(y_{2}-y_{1}\right)+Z_{1}\left(R_{2}\left(\Lambda-l_{2}^{* T} \alpha\right)\right. \\
& \left.\quad-k_{2} Z_{2}\left(f_{21}^{* T} \alpha+\Lambda g_{21}^{*}\right)\right) y_{1}=R_{1} Z_{2}\left(\Lambda+\bar{l}_{21}^{* T} \alpha-\bar{l}_{2}^{* T} \alpha\right) y_{1} .
\end{aligned}
$$

The objective now is to cancel everything but the term

$$
\left(Z_{1}(s) Z_{2}(s) \Lambda_{0}(s) R_{0}(s)\right)\left(y_{2}-y_{1}\right)=0
$$

whose dynamics are stable. In order to achieve this we have

$$
\begin{aligned}
& Z_{1}(s)\left(R_{2}(s)\left(\Lambda(s)-l_{2}^{* T} \alpha(s)\right)\right. \\
& \left.\quad-k_{2} Z_{2}(s)\left(f_{21}^{* T} \alpha(s)+\Lambda(s) g_{21}^{*}\right)\right) y_{1} \\
& \quad=R_{1}(s) Z_{2}(s)\left(\Lambda(s)+\bar{l}_{21}^{* T} \alpha(s)-\bar{l}_{2}^{* T} \alpha(s)\right) y_{1} \\
& Z_{1}(s) \\
& \quad\left(k_{2} Z_{2}(s)\left(f_{2}^{* T} \alpha(s)+g_{2}^{*} \Lambda(s)\right)+Z_{2}(s) R_{0}(s) \Lambda_{0}(s)\right. \\
& \left.\quad-k_{2} Z_{2}(s)\left(f_{21}^{* T} \alpha(s)+\Lambda(s) g_{21}^{*}\right)\right) y_{1} \\
& \quad=R_{1}(s) Z_{2}(s)\left(\Lambda(s)+\bar{l}_{21}^{* T} \alpha(s)-\bar{l}_{2}^{* T} \alpha(s)\right) y_{1} .
\end{aligned}
$$

Now remove the stable $Z_{2}(s)$ on both sides

$$
\begin{gathered}
R_{1}(s)\left(\Lambda(s)-\left(\bar{l}_{2}^{*}-\bar{l}_{21}^{*}\right)^{T} \alpha(s)\right)-k_{2} Z_{1}(s)\left(\left(f_{2}^{* T}-f_{21}^{* T}\right) \alpha(s)\right. \\
\left.-\left(g_{2}^{*}-g_{21}^{*}\right) \Lambda(s)\right)=Z_{1}(s) \Lambda_{0}(s) R_{0}(s)
\end{gathered}
$$

which gives the distributed matching condition between neighboring agents 2 and 1 .

Remark 2. The proof of Proposition 1 reveals the intrinsic meaning of the matching gains in (27), which are the ideal gains allowing agent $j$ to synchronize to agent $i$, i.e. $y_{j} \rightarrow y_{i}$ using neighboring information. Furthermore, after comparing the classical matching conditions (7) with the distributed matching conditions (27), we see that they both have solution under the same Assumptions A1-A4, M1-M2 and E1. That is, no extra assumptions are required for the solvability of (27).

Remark 3. The control (26) depends on $\Lambda(s)$ which includes $Z_{0}(s)$ : this means that some knowledge of the leader dynamics must be available in the network. This is consistent with other popular distributed protocols in literature: in fact, global knowledge of the exosystem dynamics $S$ is generally assumed in cooperative output regulation [14], while global knowledge of the leader dynamics $A_{0}$ is used in consensus [30]. The rationale is that, as soon as the leader dynamics are constant, they can be broadcast and made globally known, whereas this cannot be done for the time-varying leader signals.

Note that the matching conditions (27) introduce new gains $l_{j i}^{*}, f_{j i}^{*}, g_{j i}^{*}$ and $c_{j i}^{*}$ : since these gains (together with $l_{j}^{*}, f_{j}^{*}, g_{j}^{*}$ and $c_{j}^{*}$ ) are unknown, we propose a new adaptive law. First, let us introduce the controller for agent 2

$$
\begin{aligned}
a_{12} u_{2} & =a_{12}\left[l_{21}^{T} \frac{\alpha(s)}{\Lambda(s)} u_{1}+f_{21}^{T} \frac{\alpha(s)}{\Lambda(s)} y_{1}+g_{21} y_{1}+c_{21} u_{1}\right. \\
& \left.+l_{2}^{T} \frac{\alpha(s)}{\Lambda(s)}\left(u_{2}-u_{1}\right)+f_{2}^{T} \frac{\alpha(s)}{\Lambda(s)}\left(y_{2}-y_{1}\right)+g_{2}\left(y_{2}-y_{1}\right)\right]
\end{aligned}
$$

where $l_{21}, l_{2}, f_{21}, f_{2}, g_{21}, g_{1}$, and $c_{21}$ are the estimates for $l_{21}^{*}$, $l_{2}^{*}, f_{21}^{*}, f_{2}^{*}, g_{21}^{*}, g_{1}^{*}$, and $c_{21}^{*}$. A remark and a result follow. 
Remark 4. The controller (38) is distributed because it uses information (in particular, both states and inputs) available from neighbors. In fact, if agent 2 were connected to agent 0 , it would suffice to take a decentralized model reference adaptive controller in the form

$$
a_{02} u_{2}=a_{02}\left[l_{2}^{T} \frac{\alpha(s)}{\Lambda(s)} u_{2}+f_{2}^{T} \frac{\alpha(s)}{\Lambda(s)} y_{2}+g_{2} y_{2}+c_{2} r\right],
$$

which is not-implementable for the lack of $r$. By comparing (38) with the not-implementable (39) we note that agent 2 uses the signals (states and input) from agent 1 and the extra matching gains between agents 1 and 2 to reconstruct the reference input $r$. Similar reconstruction mechanisms appear in protocols based on distributed observer [13], [17], where, in place of neighboring inputs, agent 2 would use auxiliary observed signals from agent 1 to reconstruct the exosystem signals.

Result 2. Under Assumptions A1-A4, M1-M2 and E1, the adaptive law

$$
\begin{gathered}
\dot{\omega}_{u_{1}}=F \omega_{u_{1}}+d u_{1} \quad \dot{\omega}_{u_{21}}=F \omega_{u_{21}}+d\left(u_{2}-u_{1}\right) \\
\dot{\omega}_{y_{1}}=F \omega_{y_{1}}+d y_{1} \quad \dot{\omega}_{y_{21}}=F \omega_{y_{21}}+d\left(y_{2}-y_{1}\right) \\
a_{12} u_{2}=\theta_{2}^{T} \omega_{2} \\
\dot{\theta}_{2}=-\Gamma a_{12} e_{21} \omega_{2} \operatorname{sgn}\left(\frac{k_{2}}{k_{0}}\right)
\end{gathered}
$$

where $e_{21}=y_{2}-y_{1}, F$ and $d$ as in (10), $\Gamma>0$, and

$$
\begin{aligned}
\omega_{2} & =a_{12}\left[\begin{array}{lllllll}
\omega_{u_{1}}^{T} & \omega_{y_{1}}^{T} & y_{1} & u_{1} & \omega_{u_{21}}^{T} & \omega_{y_{21}}^{T} & y_{2}-y_{1}
\end{array}\right]^{T}, \\
\theta_{2} & =\left[\begin{array}{llllllll}
l_{21}^{T} & f_{21}^{T} & g_{21} & c_{21} & l_{2}^{T} & f_{2}^{T} & g_{2}
\end{array}\right]^{T}
\end{aligned}
$$

achieves $y_{2} \rightarrow y_{1}$ for $t \rightarrow \infty$.

Proof. To derive the adaptation law (40) of agent 2 we define

$$
\dot{\omega}_{u_{2}}=F \omega_{u_{2}}+d u_{2} \quad \dot{\omega}_{y_{2}}=F \omega_{y_{2}}+d y_{2} .
$$

Then, augmenting the state of agent 2 with the states (42), $\bar{x}_{2}=\left[\begin{array}{lll}x_{2}^{T} & \omega_{u_{2}}^{T} & \omega_{y_{2}}^{T}\end{array}\right]^{T}$ allows us to write

$$
\begin{aligned}
& \dot{\bar{x}}_{2}=\left[\begin{array}{ccc}
A_{2} & 0 & 0 \\
0 & F & 0 \\
d h_{2}^{T} & 0 & F
\end{array}\right] \bar{x}_{2}+\left[\begin{array}{c}
b_{2} \\
d \\
0
\end{array}\right] u_{2} \\
& y_{2}=\left[\begin{array}{lll}
h_{2}^{T} & 0 & 0
\end{array}\right] \bar{x}_{2}
\end{aligned}
$$

where $\left(A_{2}, B_{2}, h_{2}\right)$ is a state-space realization of agent 2 . The use of the matching conditions (37) between agent 2 and agent 1 on the terms under the parentheses here

$$
\begin{array}{ll}
\overbrace{\dot{\bar{x}}_{1}=\bar{A}_{1} \bar{x}_{1}+\bar{B}_{1} u_{1}} & \overbrace{\overline{\bar{x}}_{2}=\bar{A}_{2} \bar{x}_{2}+\bar{B}_{2} \theta_{2}^{* T} \bar{\omega}_{2}}+\bar{B}_{2} \tilde{\theta}_{2}^{T} \bar{\omega}_{2} \\
y_{1}=\bar{C}_{1}^{T} \bar{x}_{1} & y_{2}=\bar{C}_{2}^{T} \bar{x}_{2}
\end{array}
$$

with $\bar{\omega}_{2}=\omega_{2} / a_{12}$ and

$$
\begin{gathered}
\bar{A}_{2}=\left[\begin{array}{ccc}
A_{2}+b_{2} g_{2}^{*} h_{2}^{T} & b_{2} l_{2}^{* T} & b_{2} f_{2}^{* T} \\
d g_{2}^{*} h_{2}^{T} & F+d l_{2}^{* T} & d f_{2}^{* T} \\
-d h_{2}^{T} & 0 & F
\end{array}\right] \\
\bar{B}_{2}=\left[\begin{array}{c}
b_{2} \\
d \\
0
\end{array}\right], \quad \bar{C}_{2}^{T}=\left[\begin{array}{lll}
h_{2}^{T} & 0 & 0
\end{array}\right]
\end{gathered}
$$

gives rise to the following dynamics of $\tilde{x}_{21}=\bar{x}_{2}-\bar{x}_{1}$

$$
\begin{aligned}
& \dot{\tilde{x}}_{21}=\bar{A}_{2} \tilde{x}_{21}+\overline{\bar{B}}_{2} \rho_{2}^{*} \tilde{\theta}_{2}^{* T} \bar{\omega}_{2} \\
& e_{21}=\bar{C}_{2}^{T} \tilde{x}_{21}
\end{aligned}
$$

where $\overline{\bar{B}}_{2}=\bar{B}_{2} c_{2}^{*}, \rho_{2}^{*}=\frac{1}{c_{2}^{*}}$, and $\tilde{\theta}_{2}=\theta_{2}-\theta_{2}^{*}$. In addition, it can be verified that

$$
\bar{C}_{2}\left(s I-\bar{A}_{2}\right)^{-1} \overline{\bar{B}}_{2}=G_{0}(s) .
$$

In line with (19) and (21), we take the Lyapunov function

$$
V_{21}\left(\tilde{\theta}_{2}, \tilde{x}_{21}\right)=a_{12}^{2} \frac{\tilde{x}_{21}^{T} P \tilde{x}_{21}}{2}+\frac{\tilde{\theta}_{2}^{T} \Gamma^{-1} \tilde{\theta}_{2}}{2}\left|\rho_{2}^{*}\right|
$$

with $P=P^{T}>0$ satisfying the Kalman-Yakubovich Lemma

$$
\begin{aligned}
P \bar{A}_{2}+\bar{A}_{2}^{T} P & =-q q^{T}-v L \\
P \overline{\bar{B}}_{2} & =\bar{C}_{2} .
\end{aligned}
$$

The time derivative of $V_{21}$ is given by

$$
\begin{aligned}
\dot{V}_{21}=- & a_{12}^{2} \frac{\tilde{x}_{21}^{T} q q^{T} \tilde{x}_{21}}{2}-a_{12}^{2} \frac{v}{2} \tilde{x}_{21}^{T} L \tilde{x}_{21} \\
& +a_{12} \tilde{x}_{21}^{T} P \overline{\bar{B}}_{2} \rho_{2}^{*} \tilde{\theta}_{2}^{T} \omega_{2}+\tilde{\theta}_{2}^{T} \Gamma^{-1} \dot{\tilde{\theta}}_{2}\left|\rho_{2}^{*}\right| .
\end{aligned}
$$

Since $\tilde{x}_{21}^{T} P \overline{\bar{B}}_{2}=\tilde{x}_{21}^{T} \bar{C}_{2}=e_{21}$ and $\rho_{2}^{*}=\left|\rho_{2}^{*}\right| \operatorname{sgn}\left(\rho_{2}^{*}\right)$, we can delete the last two terms by choosing

$$
\dot{\tilde{\theta}}_{2}=\dot{\theta}_{2}=-\Gamma a_{12} e_{21} \omega_{2} \operatorname{sgn}\left(\rho_{2}^{*}\right)
$$

which leads to

$$
\dot{V}_{21}=-a_{12}^{2} \frac{\tilde{x}_{21}^{T} q q^{T} \tilde{x}_{21}}{2}-a_{12}^{2} \frac{v}{2} \tilde{x}_{21}^{T} L \tilde{x}_{21} .
$$

From (53) we obtain that $V_{21}$ has a finite limit, and therefore $\tilde{x}_{21}, \tilde{\theta}_{2} \in \mathscr{L}_{\infty}$. Because $\tilde{x}_{21}=\bar{x}_{2}-\bar{x}_{1} \in \mathscr{L}_{\infty}$ and $\bar{x}_{1} \in \mathscr{L}_{\infty}$, we have $\bar{x}_{2} \in \mathscr{L}_{\infty}$, which implies $x_{2}, y_{2}, \omega_{u_{21}}, \omega_{u_{2}}, \omega_{y_{21}}, \omega_{y_{2}}, \in \mathscr{L}_{\infty}$. Since from (53) we can establish that $\dot{V}_{1}$ has bounded integral, we have $\tilde{x}_{21}, e_{21} \in \mathscr{L}_{2}$. Furthermore using $\theta_{2}, \omega_{2}, \tilde{x}_{21} \in \mathscr{L}_{\infty}$ in (47) we have $e_{21}, \dot{e}_{21} \in \mathscr{L}_{\infty}$. From Barbalat's Lemma, this implies $e_{21} \rightarrow 0$ for $t \rightarrow \infty$, which concludes the proof.

\section{AdAPtive OUtPut SYNCHRONIZATION}

To arrive at the main result, the last step is to deal with the case of a follower (let us call it agent 3) aiming at synchronizing to two parent neighbors (let us call them agents 1 and 2). With a directed connection from 1 to 3 and from 2 to 3 , the digraph is described by $\mathscr{N}=\{1,2,3\}, \mathscr{E}=\{(1,3),(2,3)\}$. In order to synchronize agent 3 to agent 1 and 2 , we need to define a controller: motivated by (26), we propose

$$
\begin{gathered}
\left(a_{13}+a_{23}\right) u_{3}=a_{13} l_{31}^{T} \frac{\alpha(s)}{\Lambda(s)} u_{1}+a_{13} f_{31}^{T} \frac{\alpha(s)}{\Lambda(s)} y_{1}+a_{13} g_{31} y_{1} \\
+a_{13} c_{31} u_{1}+a_{23} l_{32}^{T} \frac{\alpha(s)}{\Lambda(s)} u_{2}+a_{23} f_{32}^{T} \frac{\alpha(s)}{\Lambda(s)} y_{2}+a_{23} g_{32} y_{2} \\
+a_{23} c_{32} u_{2}+l_{3}^{T} \frac{\alpha(s)}{\Lambda(s)} u_{a 3}+f_{3}^{T} \frac{\alpha(s)}{\Lambda(s)} e_{a_{3}}+g_{3} e_{a_{3}}
\end{gathered}
$$

where $u_{a_{3}}=a_{13}\left(u_{3}-u_{1}\right)+a_{23}\left(u_{3}-u_{2}\right), e_{a_{3}}=a_{13} e_{31}+a_{23} e_{32}$ and $e_{31}=y_{3}-y_{1}, e_{32}=y_{3}-y_{2}$; moreover, all the control gains $l_{31}, f_{31}, g_{31} c_{31}, l_{32}, f_{32}, g_{32}, c_{32}, l_{3}, f_{3}, g_{3}$ are appropriate 
estimates of ideal gains coming from the matching conditions (7) and (27). The following result holds.

Result 3. Under Assumptions A1-A4, M1-M2 and E1, the adaptive law

$$
\begin{array}{ll}
\dot{\omega}_{u_{1}}=F \omega_{u_{1}}+d u_{1} & \dot{\omega}_{y_{1}}=F \omega_{y_{1}}+d y_{1} \\
\dot{\omega}_{u_{2}}=F \omega_{u_{2}}+d u_{2} & \dot{\omega}_{y_{2}}=F \omega_{y_{2}}+d y_{2} \\
\dot{\omega}_{u_{a_{3}}}=F \omega_{u_{a_{3}}}+d u_{a_{3}} & \dot{\omega}_{e_{a_{3}}}=F \omega_{e_{a_{3}}}+d e_{a_{3}} \\
\left(a_{13}+a_{23}\right) u_{3}=\theta_{3}^{T} \omega_{3} & \dot{\theta}_{3}=-\Gamma e_{a_{3}} \omega_{3} \operatorname{sgn}\left(\frac{k_{3}}{k_{0}}\right)
\end{array}
$$

where $F$ and $d$ are as in (10), $\Gamma>0$, and

$$
\begin{aligned}
& \omega_{3}=\left[\begin{array}{llllll}
a_{13} \omega_{u_{1}}^{T} & a_{13} \omega_{y_{1}}^{T} & a_{13} y_{1} & a_{13} u_{1} & a_{23} \omega_{u_{2}}^{T} & a_{23} \omega_{y_{2}}^{T}
\end{array}\right. \\
& \left.\begin{array}{lllll}
a_{23} y_{2} & a_{23} u_{2} & \omega_{u_{a_{3}}}^{T} & \omega_{e_{a_{3}}}^{T} & e_{a_{3}}
\end{array}\right]^{T}, \\
& \theta_{3}=\left[\begin{array}{lllllllllll}
l_{31}^{T} & f_{31}^{T} & g_{31} & c_{31} & l_{32}^{T} & f_{32}^{T} & g_{32} & c_{32} & l_{3}^{T} & f_{3}^{T} & g_{3}
\end{array}\right]^{T}
\end{aligned}
$$

achieves $y_{3} \rightarrow\left(a_{13} y_{1}+a_{23} y_{2}\right) /\left(a_{13}+a_{23}\right)$ for $t \rightarrow \infty$.

Proof. As compared with $\omega_{1}$ in (10) and $\omega_{2}$ in (41), $\omega_{3}$ in (56) has two types of weights, $a_{13}$ and $a_{23}$, in view of the connection with two agents. Now, to derive the adaptation law (55) of agent 3 we have to define the dynamics of the errors $e_{31}$ and $e_{32}$. Using similar steps as (44)-(46) we find

$$
\begin{array}{ll}
\dot{\tilde{x}}_{31}=\bar{A}_{3} \tilde{x}_{31}+\bar{B}_{3} \tilde{\theta}_{31}^{T} \bar{\omega}_{31} & \dot{\tilde{x}}_{32}=\bar{A}_{3} \tilde{x}_{32}+\bar{B}_{3} \tilde{\theta}_{32}^{T} \bar{\omega}_{32} \\
e_{31}=\bar{C}_{3}^{T} \tilde{x}_{31} & e_{32}=\bar{C}_{3}^{T} \tilde{x}_{32}
\end{array}
$$

where $\bar{\omega}_{31}=\omega_{31} / a_{13}, \bar{\omega}_{32}=\omega_{32} / a_{23}$ and

$$
\begin{aligned}
\omega_{31} & =a_{13}\left[\begin{array}{lllllll}
\omega_{u_{1}}^{T} & \omega_{y_{1}}^{T} & y_{1} & u_{1} & \omega_{u_{31}}^{T} & \omega_{e_{31}}^{T} & \left(y_{3}-y_{1}\right)
\end{array}\right]^{T}, \\
\omega_{32} & =a_{23}\left[\begin{array}{lllllll}
\omega_{u_{2}}^{T} & \omega_{y_{2}}^{T} & y_{2} & u_{2} & \omega_{u_{32}}^{T} & \omega_{e_{32}}^{T} & \left(y_{3}-y_{2}\right)
\end{array}\right]^{T}, \\
\tilde{\theta}_{31} & =\left[\begin{array}{lllllll}
\tilde{l}_{31}^{T} & \tilde{f}_{31}^{T} & \tilde{g}_{31} & \tilde{c}_{31} & \tilde{l}_{3}^{T} & \tilde{f}_{3}^{T} & \tilde{g}_{3}
\end{array}\right]^{T}, \\
\tilde{\theta}_{32} & =\left[\begin{array}{lllllll}
\tilde{l}_{32}^{T} & \tilde{f}_{32}^{T} & \tilde{g}_{32} & \tilde{c}_{32} & \tilde{l}_{3}^{T} & \tilde{f}_{3}^{T} & \tilde{g}_{3}
\end{array}\right]^{T}
\end{aligned}
$$

and $\omega_{u_{31}}$ and $\omega_{u_{32}}$ are the filtered versions of $\left(u_{3}-u_{1}\right)$ and $\left(u_{3}-u_{2}\right)$, respectively. This motivates the Lyapunov function

$$
V_{321}\left(\tilde{\theta}_{2}, \tilde{x}_{21}\right)=\frac{\tilde{x}_{a_{3}}^{T} P \tilde{x}_{a_{3}}}{2}+\frac{\tilde{\theta}_{3}^{T} \Gamma^{-1} \tilde{\theta}_{3}}{2}\left|\rho_{3}^{*}\right|
$$

where $\tilde{x}_{a_{3}}=a_{13} \tilde{x}_{31}+a_{23} \tilde{x}_{32}$. The time derivative becomes, through (55)

$$
\begin{aligned}
\dot{V}_{321}=- & \frac{\tilde{x}_{a_{3}}^{T} q q^{T} \tilde{x}_{a_{3}}}{2}-\frac{v}{2} \tilde{x}_{a_{3}}^{T} L \tilde{x}_{a_{3}} \\
& +\tilde{x}_{a_{3}}^{T} P \bar{B}_{3} \rho_{3}^{*} \tilde{\theta}_{3}^{T} \omega_{3}+\tilde{\theta}_{3}^{T} \Gamma^{-1} \dot{\tilde{\theta}}_{3}\left|\rho_{3}^{*}\right| \\
=- & \frac{\tilde{x}_{a_{3}}^{T} q q^{T} \tilde{x}_{a_{3}}}{2}-\frac{v}{2} \tilde{x}_{a_{3}}^{T} L \tilde{x}_{a_{3}}
\end{aligned}
$$

where the Kalman-Yakubovich lemma analogous to (50) has led to $\tilde{x}_{a_{3}}^{T} P \overline{\bar{B}}_{3}=\tilde{x}_{a_{3}}^{T} \bar{C}_{3}=e_{a_{3}}$. Using the usual Lyapunov arguments as in Result 2 we prove $\tilde{x}_{a_{3}} \rightarrow 0$ and consequently $e_{a_{3}} \rightarrow 0$. This concludes the proof.

Remark 5. From Results 1 and 2, using the Lyapunov function $V_{1}+V_{21}$ we conclude synchronization of both agents 1 and 2 to the reference model. Furthermore, it is not difficult to conclude synchronization also when agent 1 has multiple followers.

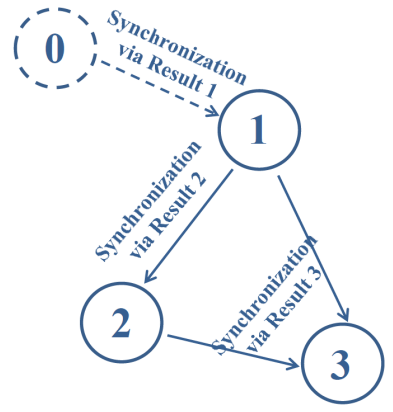

Fig. 1: Summary of Results 1, 2 and 3.

Similarly, Result 2 can be applied to any network with a directed tree topology in which each leaf has one parent and the root node has access to the reference signal $r$. In addition, by combining Results 1,2 and 3 via the Lyapunov function $V_{1}+V_{21}+V_{321}$, we have that the outputs of agents 1 and 2 converge to $y_{m}$, and consequently the output of agent 3 will converge to the average of the outputs of agents 1 and 2, i.e. to $y_{m}$ as well. This situation is summarized in Fig. 1. This reasoning is the building block to achieve synchronization over general acyclic graphs, as explained in the following.

\section{A. General acyclic case}

Consider a set of $N$ agents with transfer function

$$
y_{i}=G_{i}(s) u_{i}=k_{i} \frac{Z_{i}(s)}{R_{i}(s)} u_{i}, \quad i \in\{1, \ldots, N\}
$$

and satisfying Assumptions A1-A4. Consequently, all the matching conditions defined by (7) and (27) hold. These conditions allow us to achieve synchronization by 'cancelling' heterogeneity via appropriate gains. We make the following assumption on the communication topology.

Network assumption:

N1. The directed communication graph is acyclic and contains a directed spanning tree with the leader as the root node.

Remark 6. The absence of cycles implies the existence of a vertex permutation such that the adjacency matrix has upper triangular form. This assumption is common in protocols based on distributed input [19], [22], [39]. Extensions to cyclic and undirected networks will be discussed in Sect. V-B.

In order to synchronize all agents, we propose for the agents connected to the reference model the controller inspired by (3)

$$
a_{0 j} u_{j}=a_{0 j}\left[l_{j}^{T} \frac{\alpha(s)}{\Lambda(s)} u_{j}+f_{j}^{T} \frac{\alpha(s)}{\Lambda(s)} y_{j}+g_{j} y_{j}+c_{j} r\right]
$$

and for the agents not connected to the reference model the controller inspired by (26)

$$
\begin{aligned}
\sum_{i=1}^{N} a_{i j} u_{j}= & \sum_{i=1}^{N} a_{i j} l_{j i}^{T} \frac{\alpha(s)}{\Lambda(s)} u_{i}+\sum_{i=1}^{N} a_{i j} f_{j i}^{T} \frac{\alpha(s)}{\Lambda(s)} y_{i}+\sum_{i=0}^{N} a_{i j} g_{j i} y_{i} \\
& +\sum_{i=1}^{N} a_{i j} c_{j i} u_{i}+l_{j}^{T} \frac{\alpha(s)}{\Lambda(s)} \sum_{i=1}^{N} a_{i j}\left(u_{j}-u_{i}\right) \\
& +f_{j}^{T} \frac{\alpha(s)}{\Lambda(s)} \sum_{i=1}^{N} a_{i j}\left(y_{j}-y_{i}\right)+g_{j} \sum_{i=1}^{N} a_{i j}\left(y_{j}-y_{i}\right)
\end{aligned}
$$


where all the control gains $l_{j i}, f_{j i}, g_{j i} c_{j i}, l_{j}, f_{j}, g_{j}$ are appropriate estimates of ideal gains coming from the matching conditions (7) and (27). The following result holds.

Result 4. Under Assumptions A1-A4, M1-M2, E1 and N1, the adaptive law as in Result 1 for the agents connected to the reference model, and for the other agents the adaptive law

$$
\begin{array}{cc}
\dot{\omega}_{u_{a_{j}}}=F \omega_{u_{a_{j}}}+d \sum_{i=1}^{N} a_{i j}\left(u_{j}-u_{i}\right) & \dot{\omega}_{u_{k}}=F \omega_{u_{k}}+d u_{k} \\
\dot{\omega}_{e_{a_{j}}}=F \omega_{e_{a_{j}}}+d \sum_{i=1}^{N} a_{i j}\left(y_{j}-y_{i}\right) & \dot{\omega}_{y_{k}}=F \omega_{y_{k}}+d y_{k} \\
\sum_{i=1}^{N} a_{i j} u_{j}=\theta_{j}^{T} \omega_{j} \quad \dot{\theta}_{j}=-\Gamma \sum_{i=1}^{N} a_{i j}\left(y_{j}-y_{i}\right) \omega_{j} \operatorname{sgn}\left(\frac{k_{j}}{k_{0}}\right)
\end{array}
$$

where $k: a_{k j} \neq 0, F$ and $d$ are as in (10), $\Gamma>0$ and

$$
\begin{gathered}
\omega_{j}=\left[\begin{array}{llllll}
a_{k j} \omega_{u_{k}}^{T} & a_{k j} \omega_{y_{k}}^{T} & a_{k j} y_{k} & a_{k j} u_{k} & \ldots & k \in\{1, \ldots, N\} \\
\ldots & \omega_{u_{a_{j}}}^{T} & \omega_{e_{a_{j}}}^{T} & \sum_{i=0}^{N} a_{i j}\left(y_{j}-y_{i}\right)
\end{array}\right]^{T}, \\
\theta_{j}=\left[\begin{array}{llllll}
l_{j k}^{T} & f_{j k}^{T} & g_{j k} & c_{j k} & \ldots & k \in\{1, \ldots, N\} \\
\ldots & l_{j}^{T} & f_{j}^{T} & g_{j}
\end{array}\right]^{T}
\end{gathered}
$$

achieves $y_{i} \rightarrow y_{m}, i \in\{1, \ldots, N\}$.

Proof. The derivation of the adaptation law (64) of agent $j$ follows similar steps as in Result 3, through the error dynamics

$$
\begin{aligned}
\dot{\tilde{x}}_{j i} & =\bar{A}_{j} \tilde{x}_{j i}+\bar{B}_{j} \tilde{\theta}_{j i}^{T} \omega_{j i} \\
e_{j i} & =\bar{C}_{j}^{T} \tilde{x}_{j i}
\end{aligned}
$$

where $\tilde{\theta}_{j i}$ contains some of the components of $\tilde{\theta}_{j}$, and $\omega_{j i}$ contains some of the components of $\omega_{j}$. This motivates the following Lyapunov function

$$
V(\tilde{\theta}, \tilde{x})=\sum_{j=1}^{N} \frac{\tilde{x}_{a_{j}}^{T} P \tilde{x}_{a_{j}}}{2}+\sum_{j=1}^{N} \frac{\tilde{\theta}_{j}^{T} \Gamma^{-1} \tilde{\theta}_{j}}{2}\left|\rho_{j}^{*}\right|
$$

where $\tilde{x}_{a_{j}}=\sum_{i=0}^{N} a_{i j} \tilde{x}_{j i}$ : the time derivative gives, through (64)

$$
\dot{V}=-\sum_{j=1}^{N} \frac{\tilde{x}_{a_{j}}^{T} q q^{T} \tilde{x}_{a_{j}}}{2}-\frac{v}{2} \sum_{j=1}^{N} \tilde{x}_{a_{j}}^{T} L \tilde{x}_{a_{j}} .
$$

Using Lyapunov arguments we prove $\tilde{x}_{a_{j}} \rightarrow 0$ and using N1 we have $\tilde{x}_{j i} \rightarrow 0$ and $e_{j i} \rightarrow 0$. This concludes the proof.

Remark 7. The adaptive laws analyzed in this work are based on agents free of observation noise and unmodeled dynamics. In the presence of such phenomena, the proposed adaptive laws (64) should be replaced with robust adaptive laws in line with [38, Chap. 8]. For state synchronization, it has been shown that robust adaptive laws employing leakage, parameter projection or dynamic normalization attain robustness to bounded noises by achieving a bounded synchronization error, whose bound depends on the size of the noise [40]. In addition, it has been shown that these techniques can cope with unmodeled dynamics in the form of communication delays [41]. Similar good properties hold true for the output synchronization design proposed in this paper, but space limitations prevent us from presenting them at length.

\section{B. Handling cyclic and undirected graphs}

While the parameterization (66) is valid for acyclic graphs, it is of interest to investigate under which conditions it can be extended to graphs beyond Assumption N1, e.g. cyclic and undirected graphs. It is clear that, in the presence of cycles and undirected links, the computation of the derivative of a candidate Lyapunov function requires to sum dynamics with state-space realization $\left(\bar{A}_{j}, \overline{\bar{B}}_{j}, \bar{C}_{j}^{T}\right)$ with other dynamics with a possibly different state-space realization $\left(\bar{A}_{k}, \bar{B}_{k}, \bar{C}_{k}^{T}\right)$. A common state-space realization is a sufficient condition for making such summation possible: in adaptive literature, common state-space realizations have been shown to exist for state synchronization of systems with matched uncertainties [40], and for output synchronization using state-feedback of systems with homogeneous uncertain numerator [37, Sect. 4.2]. For output synchronization using output-feedback as in this work, a common realization of (66), call it $\left(\bar{A}_{0}, \bar{B}_{0}, \bar{C}_{0}^{T}\right)$, requires all agents to have homogeneous uncertain numerator and denominator, with possibly heterogeneous uncertain highfrequency gains [38, Sect. 6.3]. Under this condition, it is possible to show that the following holds

$$
\dot{\tilde{x}}_{a}=\left(I_{N} \otimes \bar{A}_{0}\right) \tilde{x}_{a}+\operatorname{diag}\left(\overline{\bar{B}}_{0} \tilde{\theta}_{1}^{T} \omega_{1}, \ldots, \overline{\bar{B}}_{0} \tilde{\theta}_{N}^{T} \omega_{N}\right)
$$

with $\tilde{x}_{a}=\left[\tilde{x}_{a_{1}} \cdots \tilde{x}_{a_{N}}\right]^{T}$, even in the presence of cyclic and undirected connections. At this point, the Lyapunov function

$$
V(\tilde{\theta}, \tilde{x})=\tilde{x}_{a}^{T}\left(I \otimes \frac{P}{2}\right) \tilde{x}_{a}+\sum_{j=1}^{N} \frac{\tilde{\theta}_{j}^{T} \Gamma^{-1} \tilde{\theta}_{j}}{2}\left|\rho_{j}^{*}\right|
$$

can potentially be adopted for cyclic and undirected graphs. However, in distributed input protocols, it is also necessary to guarantee that the input $u_{j}$ is well defined for all time instants: this might not be true on general graphs [39]. To explain this point, let us collect all inputs in (63) on the lefthand side, leading to $U\left[\begin{array}{lll}u_{1} & \cdots & u_{N}\end{array}\right]^{T}=\left[\begin{array}{lll}\beta_{1} & \cdots & \beta_{N}\end{array}\right]^{T}$ for an appropriate square matrix $U$. Assumption $\mathrm{N} 1$ guarantees that $U$ is always invertible: in fact, after ordering the agents in such a way that $U$ is upper triangular, we have $\operatorname{det}(U)>0$ and the control input $u_{j}$ always well defined. When assumption N1 is violated, since $U$ depends on the estimates $c_{j i}$, it is difficult if at all possible to guarantee invertibility of $U$ for every estimate. Despite this difficult analytic aspect, the simulations in the next section show that the same algorithm in Result 4 can handle networks beyond Assumption N1, with some heterogeneity in the denominator/numerator, and that $U$ turns out to be invertible at all time instants.

\section{Simulations}

Simulations are performed to illustrate the effectiveness of the approach. The simulations are carried out on the directed graph shown in Fig. 2, where the reference model is indicated as agent 0 . The agents are second-order with transfer function

$$
G_{i}(s)=\frac{s+b}{s^{2}-a_{2} s-a_{1}}
$$




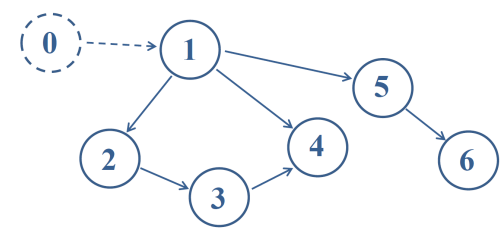

Fig. 2: The leader-follower directed communication graph.
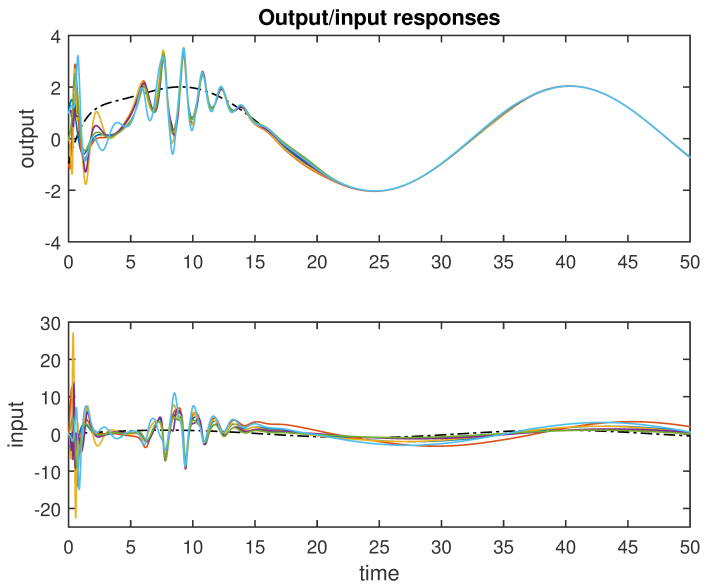

Fig. 3: Acyclic graph: input/input responses of reference model (dash-dotted) and all agents (solid).

which corresponds to the observable canonical form

$$
\begin{aligned}
\dot{x}_{i} & =\left[\begin{array}{ll}
0 & a_{1} \\
1 & a_{2}
\end{array}\right] x_{i}+\left[\begin{array}{l}
b \\
1
\end{array}\right] u_{i} \\
y & =\left[\begin{array}{ll}
0 & 1
\end{array}\right] x_{i} .
\end{aligned}
$$

The specific heterogeneous coefficients and initial conditions of each agent are reported in Table I. Except for the reference model, which is asymptotically stable, all agents are openloop unstable: in addition, all numerators are Hurwitz as per assumption. The relative degree is $n^{*}=1$ and the upper bound to the degree of the denominator is taken $n=2$. Note that the numerical values in Table I are used only to simulate the agents, while the synchronization protocol does not use the knowledge of the agents' coefficients, except $\operatorname{sgn}\left(k_{i}\right)=1$, $\forall i$. The other design parameters are taken as $\Lambda(s)=s+1$ and the adaptive gain $\Gamma_{i}$ is a diagonal matrix with gains $\gamma_{l}=1, \gamma_{f}=3, \gamma_{g}=10$, and $\gamma_{c}=1$, which correspond to the gains multiplying the coefficients $l, f, g$ and $c$, respectively. Note that dimension of $\Gamma_{i}$ depends on the dimension of $\omega_{i}$ : $\operatorname{dim}\left(\omega_{1}\right)=4, \operatorname{dim}\left(\omega_{2}\right)=\operatorname{dim}\left(\omega_{3}\right)=\operatorname{dim}\left(\omega_{5}\right)=\operatorname{dim}\left(\omega_{6}\right)=7$, and $\operatorname{dim}\left(\omega_{4}\right)=11$ (because agent 4 has 2 parents). All coupling gains are initialized to be 0 .

\begin{tabular}{l|c|c|c|c} 
& $a_{1}$ & $a_{2}$ & $b$ & $x_{0}$ \\
\hline \hline agent \#0 & -0.5 & -1 & 1 & {$\left[\begin{array}{ll}1 & -1\end{array}\right]^{\prime}$} \\
agent \#1 & -1 & 2 & 1 & {$\left[\begin{array}{ll}1 & 1\end{array}\right]^{\prime}$} \\
agent \#2 & -0.75 & 2.5 & 0.5 & {$\left[\begin{array}{rr}-1 & -1\end{array}\right]^{\prime}$} \\
agent \#3 & -1.25 & 2 & 1.25 & {$\left[\begin{array}{rr}-1 & 0\end{array}\right]^{\prime}$} \\
agent \#4 & -0.5 & 1 & 0.75 & {$\left[\begin{array}{ll}0 & 1\end{array}\right]^{\prime}$} \\
agent \#5 & -0.75 & 1 & 1.5 & {$\left[\begin{array}{ll}1 & 0\end{array}\right]^{\prime}$} \\
agent \#6 & -1.5 & 2.5 & 1 & {$\left[\begin{array}{ll}-1 & 1\end{array}\right]^{\prime}$}
\end{tabular}

TABLE I: Coefficients and initial conditions of the agents.
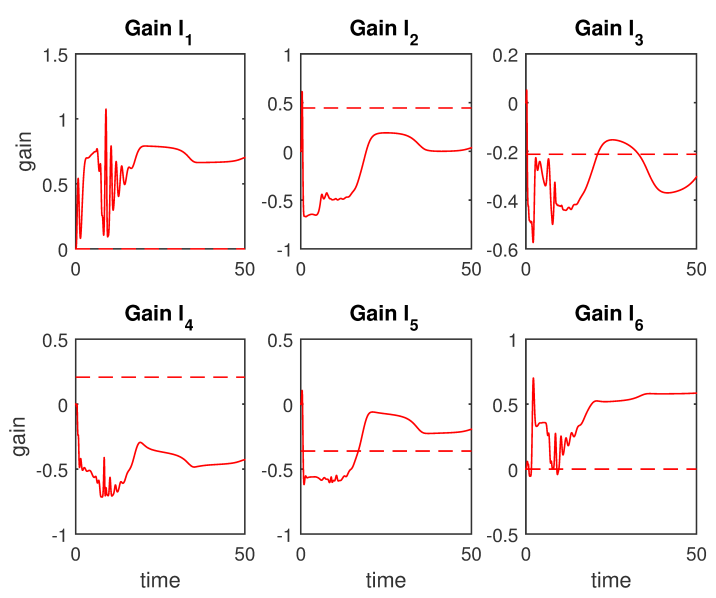

Fig. 4: Acyclic graph: Estimated (solid) vs ideal (dashed) gains.
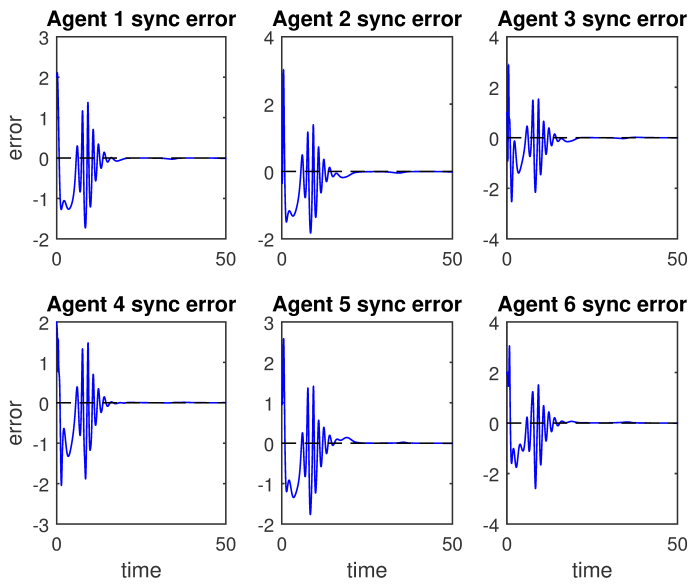

Fig. 5: Acyclic graph: Output synchronization errors $y_{i}-y_{0}$ for all agents.

The resulting synchronization for a sinusoidal reference signal is shown in Fig. 3: the outputs converge to the same behavior, while the inputs differ in view of heterogeneity. The output synchronization error can be seen in Fig. 5. Some of the estimated gains of the controllers (as compared to the ideal gains coming from the matching conditions) are shown in Fig. 4: it is noticed that not necessarily the estimated gains will converge to the ideal gains. This is a well known result in adaptive control [37]: only a persistently exciting input of sufficiently large order (not guaranteed by a single sinusoid) will lead to convergence to the ideal gains.

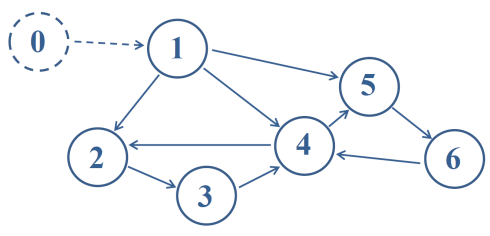

Fig. 6: Cyclic communication graph.

In order to investigate relaxations to Assumption N1, we perform additional simulations on the graph in Fig. 6, which 

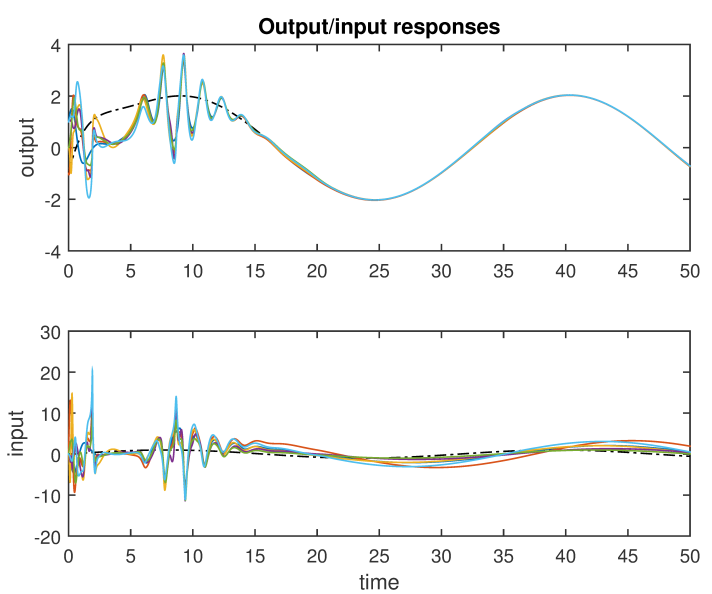

Fig. 7: Cyclic graph: Output/input responses of reference model (dash-dotted) and all agents (solid).
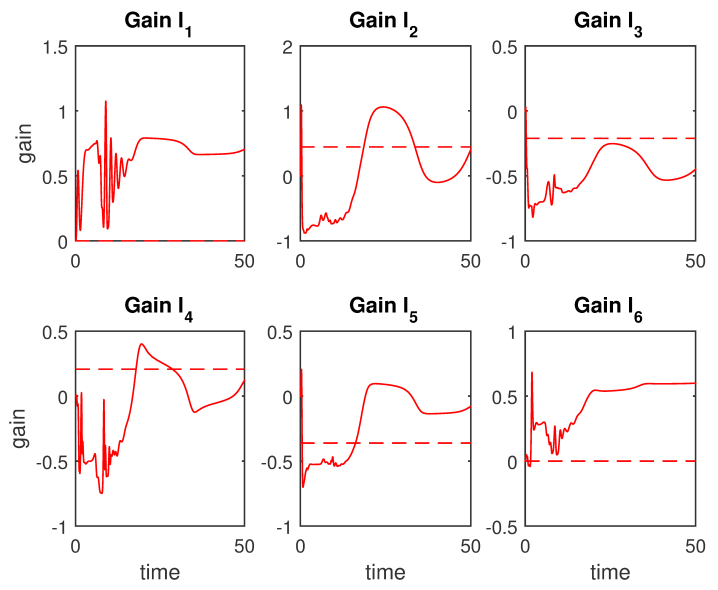

Fig. 8: Acyclic graph: Estimated (solid) vs ideal (dashed) gains.

presents two cycles among agents (cycle 2-3-4 and cycle 45-6). The resulting synchronization for a sinusoidal reference signal is shown in Fig. 7: we can see that also in this cyclic case, the outputs converge to a synchronous solution. The output synchronization error can be seen in Fig. 9. This shows that is practice the proposed algorithm can handle networks beyond Assumption N1. Some of the estimated gains of the controllers (as compared to the ideal gains coming from the matching conditions) are shown in Fig. 8: not necessarily the estimated gains will converge to the ideal gains or to the same values as in the acyclic case.

\section{CONCLUSION}

This work proposed a methodology for output synchronization of heterogeneous agents with linear and unknown dynamics. A distributed model reference adaptive control formulation was used to solve the problem. Synchronization implies matching a common behavior, and for the problem at hand, we have defined two types of matching conditions: the matching conditions between an agent and the reference model, and the matching conditions among neighboring agents. Solvability of the first set of matching conditions implies
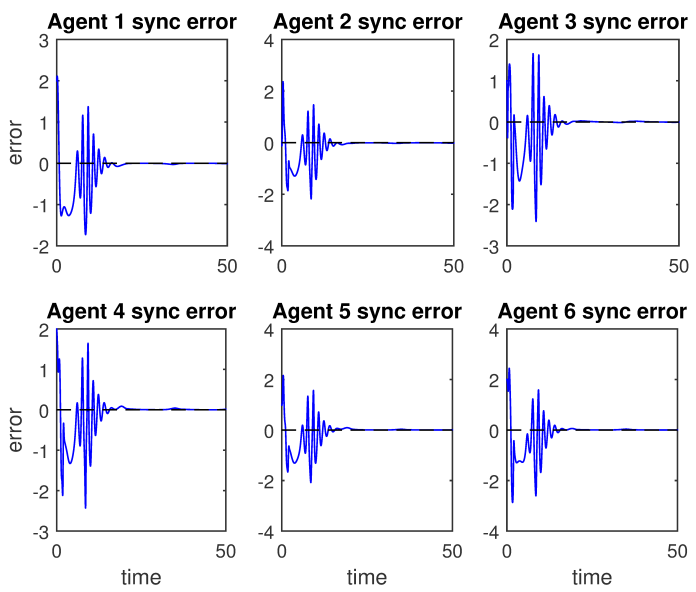

Fig. 9: Cyclic graph: Output synchronization errors $y_{i}-y_{0}$ for all agents.

solvability of the second set as well. Since all the matching gains are unknown (in view of the unknown dynamics), all the gains are adaptive and estimated using a Lyapunov-based approach.

Several research directions can stem from this work: first, since we have shown that the matching gains include both feedback and coupling gains, it is worth investigating the use of the evolution of each coupling gain to restructure the topology of the network [42]. Another related topic is the study of switching topologies via adaptive switching tools [43].

\section{REFERENCES}

[1] K.-K. Oh, M.-C. Park, and H.-S. Ahn, "A survey of multi-agent formation control," Automatica, vol. 53, pp. 424-440, 2015.

[2] F. Bullo, R. Carli, and P. Frasca, "Gossip coverage control for robotic networks: Dynamical systems on the space of partitions," SIAM Journal on Control and Optimization, vol. 50, no. 1, pp. 419-447, 2012.

[3] C. D. Korkas, S. Baldi, I. Michailidis, and E. B. Kosmatopoulos, "Occupancy-based demand response and thermal comfort optimization in microgrids with renewable energy sources and energy storage," Applied Energy, vol. 163, pp. 93-104, 2016.

[4] B. Y. Kim and H. S. Ahn, "Distributed coordination and control for a freeway traffic network using consensus algorithms," IEEE Systems Journal, vol. 10, no. 1, pp. 162-168, 2016.

[5] S. Bolouki, R. P. Malhame, M. Siami, and N. Motee, "Eminence grise coalitions: On the shaping of public opinion," IEEE Transactions on Control of Network Systems, vol. 4, no. 2, pp. 133-145, 2017.

[6] F. Dorfler and F. Bullo, "Synchronization in complex networks of phase oscillators: A survey," Automatica, vol. 50, no. 6, pp. 1539-1564, 2014.

[7] L. F. R. Turci, P. De Lellis, E. E. N. Macau, M. di Bernardo, and M. M. R. Simões, "Adaptive pinning control: A review of the fully decentralized strategy and its extensions," The European Physical Journal Special Topics, vol. 223, no. 13, pp. 2649-2664, 2014.

[8] R. Olfati-Saber and R. M. Murray, "Consensus problems in networks of agents with switching topology and time-delays," IEEE Transactions on Automatic Control, vol. 49, no. 9, pp. 1520-1533, 2004.

[9] W. Ren and R. W. Beard, "Consensus seeking in multiagent systems under dynamically changing interaction topologies," IEEE Transactions on Automatic Control, vol. 50, no. 5, pp. 655-661, 2005.

[10] F. Fagnani and P. Frasca, Introduction to Averaging Dynamics over Networks. (Lecture Notes in Control and Information Sciences), Springer, 2017.

[11] Z. Li, Z. Duan, G. Chen, and L. Huang, "Consensus of multiagent systems and synchronization of complex networks: A unified viewpoint," IEEE Transactions on Circuits and Systems I: Regular Papers, vol. 57, no. 1, pp. 213-224, 2010. 
[12] A. Jadbabaie, J. Lin, and A. S. Morse, "Coordination of groups of mobile autonomous agents using nearest neighbor rules," IEEE Transactions on Automatic Control, vol. 48, no. 6, pp. 988-1001, 2003.

[13] Y. Su and J. Huang, "Cooperative output regulation of linear multi-agent systems," IEEE Transactions on Automatic Control, vol. 57, no. 4, pp. 1062-1066, 2012.

[14] P. Wieland, R. Sepulchre, and F. Allgower, "An internal model principle is necessary and sufficient for linear output synchronization," Automatica, vol. 47, no. 5, pp. 1068-1074, 2011.

[15] A. L. Fradkov and A. Y. Markov, "Adaptive synchronization of chaotic systems based on speed gradient method and passification," IEEE Transactions on Circuits and Systems I: Fundamental Theory and Applications, vol. 44, no. 10, pp. 905-912, 1997.

[16] T. E. Gibson, "Adaptation and synchronization over a network: Asymptotic error convergence and pinning," in 2016 IEEE 55th Conference on Decision and Control (CDC), 2016, pp. 2969-2974.

[17] M. Lu and L. Liu, "Distributed feedforward approach to cooperative output regulation subject to communication delays and switching networks," IEEE Transactions on Automatic Control, vol. 62, no. 4, pp. 1999-2005, 2017.

[18] W. Ren, "Multi-vehicle consensus with a time-varying reference state," Systems \& Control Letters, vol. 56, no. 78, pp. 474-483, 2007.

[19] S. Khoo, L. Xie, S. Zhao, and Z. Man, "Multi-surface sliding control for fast finite-time leader-follower consensus with high order SISO uncertain nonlinear agents," International Journal of Robust and Nonlinear Control, vol. 24, no. 16, pp. 2388-2404, 2014.

[20] Y. A. Harfouch, S. Yuan, and S. Baldi, "An adaptive approach to cooperative longitudinal platooning of heterogeneous vehicles with communication losses," in 20th IFAC World Congress, July 9th-14th, Toulouse, France, 2017, pp. 1382-1387.

[21] J. Ploeg, N. van de Wouw, and H. Nijmeijer, " $l_{p}$ string stability of cascaded systems: Application to vehicle platooning," IEEE Transactions on Control Systems Technology, vol. 22, pp. 786-793, 2014.

[22] Y. A. Harfouch, S. Yuan, and S. Baldi, "An adaptive switched control approach to heterogeneous platooning with inter-vehicle communication losses," IEEE Transactions on Control of Network Systems, 2017, in press, available online.

[23] F. Chen, Y. Cao, and W. Ren, "Distributed average tracking of multiple time-varying reference signals with bounded derivatives," IEEE Transactions on Automatic Control, vol. 57, no. 12, pp. 3169-3174, 2012.

[24] Z. Li, X. Liu, W. Ren, and L. Xie, "Distributed tracking control for linear multiagent systems with a leader of bounded unknown input," IEEE Transactions on Automatic Control, vol. 58, no. 2, pp. 518-523, 2013.

[25] Z. Li and Z. Ding, "Distributed adaptive consensus and output tracking of unknown linear systems on directed graphs," Automatica, vol. 55, pp. $12-18,2015$.

[26] Z. Ding and Z. Li, "Distributed adaptive consensus control of nonlinear output-feedback systems on directed graphs," Automatica, vol. 72, pp. 46-52, 2016.

[27] Z. Li, M. Z. Q. Chen, and Z. Ding, "Distributed adaptive controllers for cooperative output regulation of heterogeneous agents over directed graphs," Automatica, vol. 68, pp. 179-183, 2016.

[28] G. S. Seyboth, W. Ren, and F. Allgower, "Cooperative control of linear multi-agent systems via distributed output regulation and transient synchronization," Automatica, vol. 68, pp. 132-139, 2016.

[29] J. Mei, W. Ren, and J. Chen, "Distributed consensus of second-order multi-agent systems with heterogeneous unknown inertias and control gains under a directed graph," IEEE Transactions on Automatic Control, vol. 61, no. 8, pp. 2019-2034, 2016.

[30] Z. Li, W. Ren, X. Liu, and L. Xie, "Distributed consensus of linear multiagent systems with adaptive dynamic protocols," Automatica, vol. 49, no. 7, pp. 1986-1995, 2013.

[31] Z. Li, W. Ren, X. Liu, and M. Fu, "Consensus of multi-agent systems with general linear and Lipschitz nonlinear dynamics using distributed adaptive protocols," IEEE Transactions on Automatic Control, vol. 58, no. 7, pp. 1786-1791, 2013.

[32] G. S. Seyboth, D. V. Dimarogonas, K. H. Johansson, P. Frasca, and F. Allgower, "On robust synchronization of heterogeneous linear multiagent systems with static couplings," Automatica, vol. 53, pp. 392-399, 2015.

[33] H. Liu, C. De Persis, and M. Cao, "Robust decentralized output regulation with single or multiple reference signals for uncertain heterogeneous systems," International Journal of Robust and Nonlinear Control, vol. 25, no. 9, pp. 1399-1422, 2015.

[34] J. Mei, W. Ren, B. Li, and G. Ma, "Distributed containment control for multiple unknown second-order nonlinear systems with application to networked Lagrangian systems," IEEE Transactions on Neural Networks and Learning Systems, vol. 26, no. 9, pp. 1885-1899, 2015.

[35] S. Ghapani, J. Mei, W. Ren, and Y. Song, "Fully distributed flocking with a moving leader for Lagrange networks with parametric uncertainties," Automatica, vol. 67, pp. 67-76, 2016.

[36] G. Tao, "Multivariable adaptive control: A survey," Automatica, vol. 50, no. 11 , pp. 2737 - 2764, 2014.

[37] _- Adaptive Control Design and Analysis. Wiley, 2003.

[38] P. Ioannou and J. Sun, Robust Adaptive Control. Dover Publications, 2012.

[39] W. Wang, C. Wen, J. Huang, and Z. Li, "Hierarchical decomposition based consensus tracking for uncertain interconnected systems via distributed adaptive output feedback control," IEEE Transactions on Automatic Control, vol. 61, no. 7, pp. 1938-1945, 2016.

[40] S. Baldi and P. Frasca, "Adaptive synchronization of unknown heterogeneous agents: an adaptive virtual model reference approach," Journal of The Franklin Institute, Special Issue: Modeling, Analysis and Control of Networked Autonomous Agents, 2018, in press, available online.

[41] G. Lymperopoulos and P. Ioannou, "Adaptive control of networked distributed systems with unknown interconnections," in 2016 IEEE 55th Conference on Decision and Control (CDC), 2016, pp. 3456-3461.

[42] P. De Lellis, M. di Bernardo, F. Garofalo, and M. Porfiri, "Evolution of complex networks via edge snapping," IEEE Transactions on Circuits and Systems I: Regular Papers, vol. 57, no. 8, pp. 2132-2143, 2010.

[43] S. Yuan, B. De Schutter, and S. Baldi, "Adaptive asymptotic tracking control of uncertain time-driven switched linear systems," IEEE Transactions on Automatic Control, vol. 62, no. 11, pp. 5802-5807, 2017.

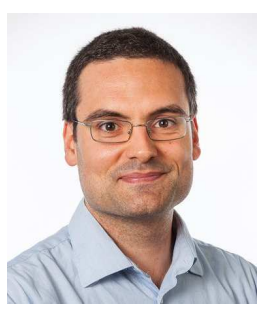

Simone Baldi (M'16) received the B.Sc., M.Sc. and $\mathrm{Ph} . \mathrm{D}$. degree from University of Florence, Italy, in 2005, 2007, and 2011 respectively. He is currently Assistant Professor at the Delft Center for Systems and Control, Delft University of Technology. Previously, he held post-doc researcher positions at University of Cyprus, and at Information Technologies Institute, CERTH, Thessaloniki. His research interests include adaptive and switched systems with applications in networked control and multi-agent systems.

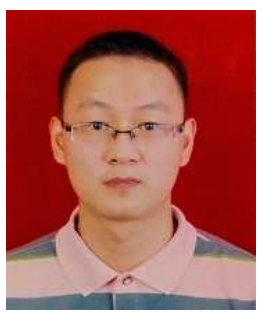

Shuai Yuan received the B.Sc. and M.Sc. degree in Mechanical Science and Engineering from Harbin Institute of Technology, Huazhong University of Science and Technology, China, in 2011 and 2014 respectively. $\mathrm{He}$ is currently a $\mathrm{PhD}$ candidate at the Delft Center for Systems and Control, Delft University of Technology. His research interests include adaptive systems and switched systems.

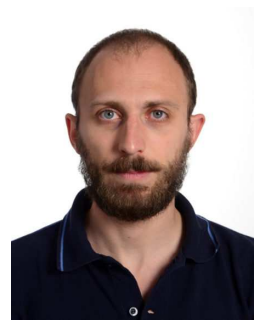

Paolo Frasca (M'13) received the Ph.D. degree in Mathematics for Engineering Sciences from Politecnico di Torino, Torino, Italy, in 2009. Between 2008 and 2013, he held research and visiting positions at the University of California, Santa Barbara (USA), at the IAC-CNR (Rome, Italy), at the University of Salerno (Italy), and at the Politecnico di Torino. From 2013 to 2016, he was an Assistant Professor at the University of Twente in Enschede, the Netherlands. In October 2016 he joined the CNRS as a Researcher: he is currently affiliated with GIPSAlab in Grenoble, France. His research interests are in the theory of network systems and cyber-physical systems, with applications to robotic, sensor, infrastructural, and social networks. He is a recipient of the 2013 SIAG/CST Best SICON Paper Prize. Dr. Frasca has served in the Conference Editorial Boards of several events, including IEEE CDC, ACC, ECC, MTNS, IFAC NecSys, and is currently serving as Associate Editor for the International Journal of Robust and Nonlinear Control, the Asian Journal of Control, and the IEEE Control Systems Letters. 